\title{
Constructal design aplicado às cavidades convectivas inseridas em um sólido cilíndrico
}

\author{
Constructal design applied to convective cavities inserted into a cylindrical solid body
}

\author{
E. S. D. Estrada ${ }^{1 *}$; G. V. Gonzales ${ }^{2}$; E. D. dos Santos ${ }^{3}$; L. A. Isoldi ${ }^{3}$; L. A. O. \\ Rocha $^{4}$ \\ ${ }^{1}$ Centro de Ciências Computacionais, Universidade Federal do Rio Grande, 96203-900, Rio Grande-RS, Brasil \\ ${ }^{2}$ Programa de Pós-Graduação em Modelagem Computacional, Universidade Federal do Rio Grande, 96203-900, \\ Rio Grande-RS, Brasil \\ ${ }^{3}$ Escola de Engenharia, Universidade Federal do Rio Grande, 96203-900, Rio Grande-RS, Brasil \\ ${ }^{4}$ Departamento de Engenharia Mecânica, Universidade Federal do Rio Grande Sul, 90040-060, Porto Alegre-RS, \\ Brasil \\ *emanuelestrada@gmail.com
}

(Recebido em 22 de setembro de 2014; aceito em 29 de dezembro de 2014)

\begin{abstract}
Este trabalho aplica Constructal design no estudo numérico da geometria de cavidades em contato com um fluido com coeficiente de transferência de calor constante que são inseridas em um sólido cilíndrico. O objetivo principal do trabalho é minimizar a máxima temperatura entre o sólido e o ambiente através da variação da geometria da cavidade. A geração interna de calor é distribuída uniformemente no sólido que tem condições adiabáticas nas superfícies externas. O volume total e o volume das cavidades são fixos. As cavidades são retangulares, com razões variáveis. A geometria otimizada e desempenho são apresentados como funções da razão entre o volume das cavidades e o volume total, o número de cavidades e o parâmetro adimensional que representa a transferência de calor por convecção, $\lambda$. Os resultados principais mostram que para um número fixo de cavidades, $\phi \mathrm{c}$ e o parâmetro adimensional $\lambda$, existe um número ideal de cavidades que minimiza a temperatura máxima e este número ideal de cavidades aumenta, em geral, a medida que $\phi_{\mathrm{c}}$ e $\lambda$ aumentam
\end{abstract}

Palavras-chave: cavidades convecivas; constructal design; geração de calor

This work applies Constructal design to study numerically the geometry of cavities bathed by a fluid with constant heat transfer coefficient that are intruded into a cylindrical solid body. The objective is to minimize the maximum excess of temperature between the solid body and the ambient by morphing the cavity geometry. Internal heat generating is distributed uniformly into the solid body, which has adiabatic conditions on the outer surfaces. The total volume and the volume of the cavities are fixed. The cavities are rectangular with variable aspect ratio. The optimized geometry and performance are reported as functions of the ratio between the volume of the cavities and the total volume, the number of cavities and the dimensionless parameter that accounts for the convective heat transfer, $\lambda$. The main results indicate that for fixed number of cavities, $\phi_{c}$, and dimensionless parameter $\lambda$, there are an optimal number of cavities that minimizes the maximum excess of temperature and this optimal number of cavities in general increases as $\phi_{c}$ and $\lambda$ increases.

Keywords: Constructal design; convective cavities; heat generating

\section{INTRODUÇÃO}

A lei Constructal do design e evolução [1] tem sido usada para determinar as melhores configurações que facilitam o acesso de suas correntes em sistemas de fluxo de tamanho finito. Esta lei da física explica o fenômeno de configuração de fluxo em sistemas animados e inanimados [2-4]. Uma grande quantidade de trabalhos produzidos relacionados à lei Constructal são apresentados e documentados recorrentemente na literatura [5-8]. Um exemplo recente de importante contribuição, desta área que vem crescendo, é o estudo da evolução de aviões sob a luz da lei Constructal [9].

O Constructal design é o método que é usado para aplicar lei Constructal [4]. Tem sido aplicado quando a geometria dos sistemas de fluxo de tamanho finito é desconhecida. Exemplos 
da aplicabilidade do método são apresentados na descoberta de novos designs na eletrônica, células de combustível e redes para o transporte de pessoas, mercadorias e informações [10-16].

Um interesse particular surgiu na busca de melhores formas de aumentar a transferência de calor em aletas e cavidades, isto é, aletas invertidas ou negativas. Neste sentido o Constructal design tem sido usado com sucesso para encontrar melhores arquiteturas formas simples de $\mathrm{T} e$ Y, bem como no arranjo complexo de configurações de aletas, como por exemplo, aletas arranjadas e, forma de T-Y [17-19]. Cavidades em forma de C, T, Y, duplo Y, H e T-Y também foram estudadas em [20-24]. As melhores formas de cavidades retangulares isotérmicas inseridos num corpo cilíndrico, também foram calculadas para vários números de cavidades em [25].

Este trabalho numérico aplica o método Constructal para encontrar as melhores configurações de cavidades convectivas retangulares inseridas em um sólido cilíndrico com geração interna de calor uniforme. As cavidades estão em contato com um fluido com coeficiente de transferência de calor constante. As superfícies externas do sólido são adiabáticas. $\mathrm{O}$ volume total e o volume das cavidades são constantes, mas a razão de aspecto da cavidade podem variar. $\mathrm{O}$ objetivo é minimizar máxima temperatura entre o sólido e o ambiente. O número de cavidades, a razão entre o volume das cavidades e o volume total e o parâmetro adimensional representando a transferência de calor por convecção na superfície das cavidades são os parâmetros do problema.

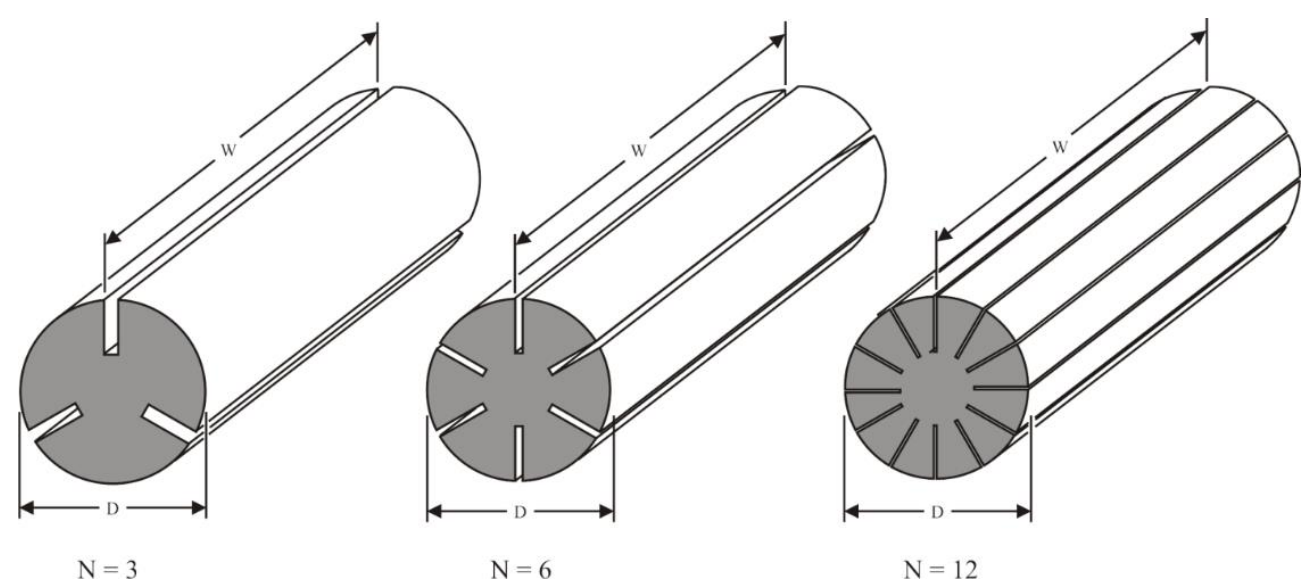

Figura 1: Exemplo de cavidades convectivas retangulares inseridas em um sólido cilíndrico

\section{GEOMETRIA DO SÓLIDO CILÍNDRICO}

A metodologia deve ser descrita com as informações necessárias para permitir a repetição do estudo por outro pesquisador.

Considere o sólido cilíndrico na Figura 1. Existe um número $N$ de cavidades convectivas retangulares inseridas corpo. O sólido é isotrópico com uma condutibilidade térmica constante k. Ele gera um calor uniforme à uma taxa volumétrica q'" $\left(\mathrm{W} / \mathrm{m}^{3}\right)$. As superfícies externas do corpo com geração de calor são perfeitamente isoladas. A corrente de calor gerado (q"'A) é removida por convecção através das paredes da cavidade. A terceira dimensão (W) é suficientemente longa, em comparação com o raio $\mathrm{R}$ do volume ocupado pelo corpo, por conseguinte, é assumida uma configuração bidimensional, como mostrado na Figura 2.

O objetivo da análise é determinar a geometria ideal que é caracterizada pela mínima temperatura máxima $\left(\mathrm{T}_{\max }-\mathrm{T}_{\infty}\right) /(\mathrm{q} " \mathrm{q} / \mathrm{k})$, para diferentes valores de fluxos convectivos aplicados às superfícies das cavidades. De acordo com a concepção Constructal [4], esta optimização pode ser submetida a duas restrições, denominadas, a restrição de área total,

$$
\mathrm{A}=\pi \mathrm{R}^{2}
$$

e a restrição de área da cavidade, 


$$
\mathrm{A}_{\mathrm{c}}=\mathrm{NH}_{0} \mathrm{~L}_{0}
$$

onde $N$ é o número de cavidades inseridas no sólido. Esta equação pode ser substituída pela fração de área ocupada pelas cavidades

$$
\phi_{\mathrm{c}}=\frac{\mathrm{A}_{\mathrm{c}}}{\mathrm{A}}
$$

A análise que determina a resistência térmica global adimensional como uma função da geometria consiste em resolver numericamente a equação da condução do calor para o domínio do sólido,

$$
\frac{\partial^{2} \theta}{\partial \tilde{\mathbf{x}}^{2}}+\frac{\partial^{2} \theta}{\partial \tilde{\mathbf{y}}^{2}}+1=0
$$

onde as variáveis adimensionais são

$$
\theta=\frac{\mathrm{T}-\mathrm{T}_{\infty}}{\mathrm{q} " \mathrm{~A} / \mathrm{k}}
$$

$\mathrm{e}$

$$
\tilde{\mathrm{x}}, \tilde{\mathrm{y}}, \tilde{\mathrm{H}}_{0}, \tilde{\mathrm{L}}_{0}, \tilde{\mathrm{R}}=\frac{\mathrm{x}, \mathrm{y}, \mathrm{H}_{0}, \mathrm{~L}_{0}, \mathrm{R}}{\mathrm{A}^{1 / 2}}
$$

As superfícies externas são isoladas com condição de contorno

$$
\frac{\partial \theta}{\partial \widetilde{\mathbf{n}}}=0
$$

As condições de contorno nas superfícies das cavidades são dadas por

$$
\frac{\partial \theta}{\partial \tilde{n}}=-\lambda \theta
$$

onde $n$ é a coordenada normal à superfície interna da cavidade e o parâmetro $\lambda$ que aparece na equação (8) é o mesmo adotado em [26] e similar ao usado nos estudos [17-19]. O parâmetro $\lambda$ é dado por:

$$
\lambda=\frac{\mathrm{hA}^{1 / 2}}{\mathrm{k}}
$$

A forma adimensional das Equações (1) e (3) são

$$
\begin{aligned}
& 1=\pi \tilde{\mathrm{R}}^{2} \\
& \phi_{\mathrm{c}}=\mathrm{NH}_{0} \tilde{\mathrm{L}}_{0}
\end{aligned}
$$


O objetivo principal é minimizar a temperatura máxima, $\theta_{\max }$, dada por:

$$
\theta_{\max }=\frac{\mathrm{T}_{\max }-\mathrm{T}_{\infty}}{\mathrm{q} " \mathrm{~A} / \mathrm{k}}
$$

Cabe destacar que o presente trabalho não é obtido para um problema conjugado de transferência de calor, mas somente para condução em um sólido (com geração de calor em várias magnitudes relativas impostas a condição de contorno convectiva).

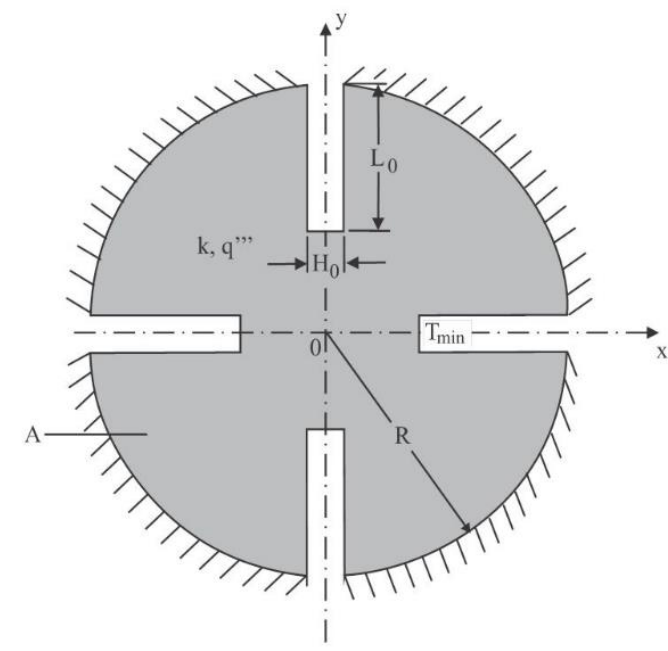

Figura 2: Modelo bidimensional do sólido cilíndrico com inserção de cavidades convectivas retangulares.

\section{MODELO NUMÉRICO}

O trabalho numérico consiste em determinar o campo de temperatura em um grande número de configurações do tipo mostrado na Figura 2. A função definida pela Equação (12) é determinada numericamente, resolvendo a Equação (14) para o campo de temperatura em cada configuração assumida $\left(N, H_{0} / L_{0}\right)$ e, assim, calculando $\theta_{\max }$ de forma a identificar onde este pode ser minimizado pela variação da configuração. Neste sentido, a Equação (4) é resolvida por uma abordagem de elementos finitos, usando uma malha triangular não-estruturada e nãoregular. O desenvolvimento da solução foi feito em ambiente MATLAB com o auxílio da toolbox PDE (partial differential equations). A malha foi variada a cada nova geometria. Assim, o tamanho apropriado da malha é determinado por refinamentos sucessivos, quadriplicando o número de elementos da malha a cada novo refinamento, até o critério de $\left(\theta_{\max }^{\mathrm{j}}-\theta_{\max }^{\mathrm{j}+1}\right) / \theta_{\max }^{\mathrm{j}} \mid<5 \times 10^{-4}$ ser atendido. Sendo $\theta_{\max }^{\mathrm{j}}$ a temperatura máxima calculada usando o tamanho de malha atual e $\theta_{\max }^{\mathrm{j}+1}$ a temperatura máxima usada no próximo refinamento de malha. A Tabela 1 exemplifica como a independência de malha foi adquirida, realizado testes para uma configuração onde $N=4, \phi_{c}=0.1, H_{0} / L_{0}=0.149$ e $\lambda=1000$. Os resultados foram obtidos usando uma malha que variou entre 100 e 10000 elementos triangulares. A precisão da abordagem numérica é validada pela comparação dos resultados com os encontrados para um mesmo caso usando o software COMSOL [28]. Ambos resultados apresentaram uma boa concordância. 
Tabela 1: Teste de independência de malha $\left(N=4, \phi_{c}=0,1, H_{0} / L_{0}=0,149, \lambda=1000\right)$.

\begin{tabular}{ccc}
\hline Número de elementos & $\theta_{\max }^{\mathrm{j}}$ & $\left|\left(\theta_{\max }^{\mathrm{j}}-\theta_{\max }^{\mathrm{j}+1}\right) / \theta_{\max }^{\mathrm{j}}\right|$ \\
\hline 300 & 0.04156 & $9.62 \times 10^{-4}$ \\
1,200 & 0.04152 & $2.41 \times 10^{-4}$ \\
4,800 & 0.04153 & \\
\hline
\end{tabular}

\section{GEOMETRIA ÓTIMA}

A Figura 3 mostra o efeito da razão $H_{0} / L_{0}$ sobre a de temperatura máxima, $\theta_{\max }$, quando o número de cavidades e parâmetro $\lambda$ são $N=4$ e $\lambda=0,1$, respectivamente, para vários valores da fração de cavidade $\phi_{c}$. Para todos $\phi_{c}$, a temperatura máxima uma vez minimizada, $\left(\theta_{\max }\right)_{\mathrm{m}}$, é obtida para os valores mínimos de $H_{0} / L_{0}$, ou seja, quando as cavidades têm a maior inserção no disco cilíndrico. Quando a grandeza $\phi_{c}$ diminui, as cavidades penetram mais no interior do sólido com o propósito de melhorar a troca de calor entre as superfícies das cavidades e o sólido. $\mathrm{O}$ efeito de $H_{0} / L_{0}$ sobre $\theta_{\max }$, obtido para $\lambda=0,1$, é diferente do observado no estudo [25] (otimização geométrica de cavidades retangulares isotérmicas) onde foram encontradas razões ótimas de valores intermediários para $H_{0} / L_{0}$ e diferentes valores de $\phi_{c}$, ou seja, o valor mínimo da razão $H_{0} / L_{0}$ calculado para $\lambda=0,1$, está localizado no extremo inferior do domínio. Portanto, os resultados mostram que as alterações/adaptações geométricas em sistemas de fluxo pode ser causada pelo efeito de vizinhança (condições de contorno).

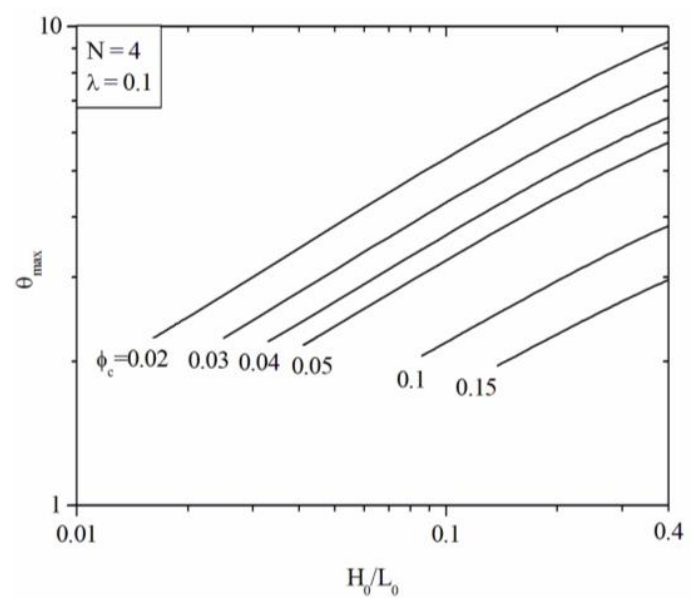

Figura 3: Otimização da temperatura máxima em função da razão $H_{0} / L_{0}$ para $N=4, \lambda=0,1$ e diferentes valores de $\phi_{c}$.

Com a finalidade de investigar a influência do coeficiente de transferência de calor, representado aqui pelo parâmetro $\lambda$, através da optimização da temperatura máxima $\left(\theta_{\max }\right)$ como função da razão $H_{0} / L_{0}$, a Figura 4 apresenta o efeito de cinco diferentes valores de parâmetro $\lambda$ : $\lambda=0,1,1,10,100$ e 1000 . Para todas as simulações, os parâmetros $N$ e $\phi_{c}$ são mantidos fixo ( $N$ $=4$ e $\left.\phi_{c}=0,1\right)$. Para os menores valores de $\lambda$ avaliados $(\lambda=0,1$ e 1$)$ a temperatura máxima uma vez minimizada, $\left(\theta_{\max }\right)_{\mathrm{m}}$, é obtida para as razões mínimas de $H_{0} / L_{0}$, como pode ser observado na Figura 3. Conforme a magnitude de $\lambda$ aumenta, o melhor desempenho agora é alcançado para razões intermediárias de $H_{0} / L_{0}$. A Figura 4 mostra também que o aumento de $\lambda$ não conduz necessariamente à melhor performance térmica do calor gerado no disco em cada intervalo de $H_{0} / L_{0}$ avaliado. Por exemplo, a forma ideal obtida para $\lambda=100$ tem um desempenho superior ao 
que foi alcançado por $H_{0} / L_{0}>0,26$ e $\lambda=1000$. Consequentemente, a geometria é extremamente importante para melhorar o desempenho de sistemas de fluxo térmico. Além disso, a racionalização do design em sistemas de fluxo pode compensar o uso valores pequenos do parâmetro $\lambda$ neste tipo de problema. $\mathrm{O}$ mesmo procedimento realizado na Figura 3 para $N=4 \mathrm{e}$ $\lambda=0.1$ é repetido para os outros valores de $\lambda$ estudados.

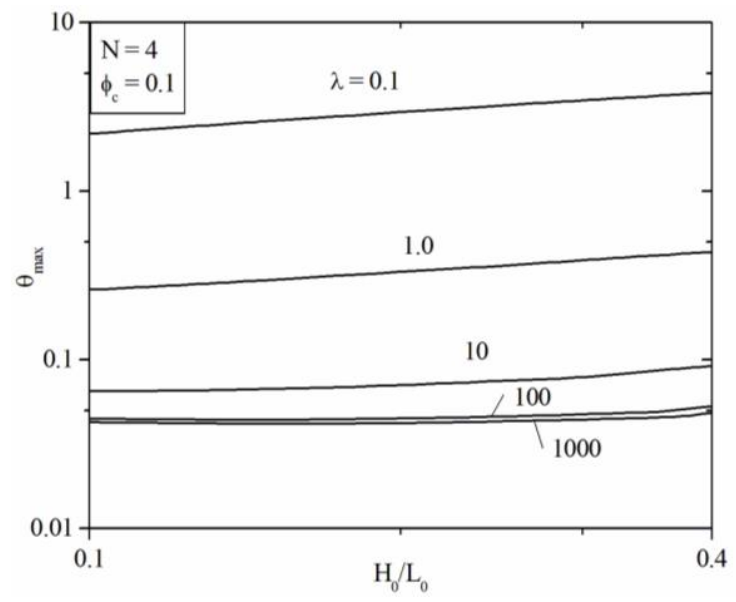

Figure 4: Otimização da temperatura máxima em função da razão $H_{0} / L_{0}$ para $N=4, \phi_{c}=0,1 e$ diferentes valores de $\lambda$

A temperatura máxima uma vez minimizada, $\left(\theta_{\max }\right)_{\mathrm{m}}$, e a sua respectiva forma ótima, $\left(H_{0} / L_{0}\right)_{\text {o }}$, obtida para cada valor de $\phi_{c}$ e três valores diferentes de $\lambda(\lambda=0,1,10$ e 1000) são encontradas resumidas nas Figuras. 5 e 6, respectivamente. Como esperado, o aumento de $\lambda$ conduz a uma melhora do desempenho térmico para todos os valores de $\phi_{c}$. A Figura 5 mostra que, para os três valores de $\lambda$, a temperatura máxima uma vez minimizada $\left(\theta_{\max }\right)_{\mathrm{m}}$ diminui com $\mathrm{o}$ aumento de $\phi_{c}$. Esta queda é mais evidente para o caso onde $\lambda=0,1$ do que para os outros dois valores de $\lambda\left(\lambda=10\right.$ e 1000), ou seja, os resultados mostram uma maior dependência de $\phi_{c}$ para valores menores de $\lambda$. Para $\lambda=0,1$ e $N=4$ é notado também uma menor diminuição de $\left(\theta_{\max }\right)_{\mathrm{m}}$ para $\phi_{c} \leq 0,045$ do que o observado para $\phi_{c} \geq 0,045$. Para este caso específico $(N=4$ e $\lambda=0,1)$ o parâmetro $\phi_{c}$ tem uma influência maior sobre o comportamento térmico do problema quando $\phi_{c} \geq 0,045$. A Figura 6 mostra o efeito de $\phi_{c}$ em relação a razão uma vez otimizada $\left(H_{0} / L_{0}\right)_{o}$ para os três diferentes valores de $\lambda$ acima mencionado. Em geral, ocorre um aumento de $\left(H_{0} / L_{0}\right)$ quando também ocorre o aumento do parâmetro $\phi_{c}$, ou seja, a inserção das cavidades para o disco reduz devido à interferência física entre as cavidades. Os resultados também mostraram um aumento de $\left(H_{0} / L_{0}\right)_{0}$ para todos os valores de $\phi_{c}$, junto com o aumento do parâmetro $\lambda$, com exceção dos parâmetros $\lambda=0,1$ e 10 quando $\phi_{c}<0,045$. No extremo inferior de $\lambda$, o fluxo de calor nas cavidades é baixo sendo necessário uma inserção mais profunda dentro do disco, com o propósito de evitar a geração de um ponto de calor na região central do domínio. À medida que a magnitude de $\lambda$ aumenta, a capacidade das cavidades de remover a energia do sólido também é aumentada. Como consequência, as cavidades diminuem a sua inserção no interior do domínio sólido com a finalidade de distribuir mais uniformemente os pontos de calor em cinco pontos (para $N=4$ ): um no centro do sólido e quatro entre as cavidades adjacentes na periferia do disco. O mesmo procedimento realizado na Figura. 4 para a avaliação de $\theta_{\max }$ como uma função de $H_{0} / L_{0}$ para vários valores de $\lambda, N=4$ e $\phi_{c}=0,1$, é realizado aqui tendo em conta vários valores de $N(4 \leq N \leq 32)$. Os resultados óptimos obtidos neste procedimento levou à construção das Figuras 7 e 8 . A Figura 7 mostra o efeito do parâmetro $\lambda$ sobre $\left(\theta_{\max }\right)_{\mathrm{m}}$, para os vários valores de $N$ investigados e a Figura 8 mostra o efeito do parâmetro $\lambda$ sobre $\left(H_{0} / L_{0}\right)_{\circ}$. 


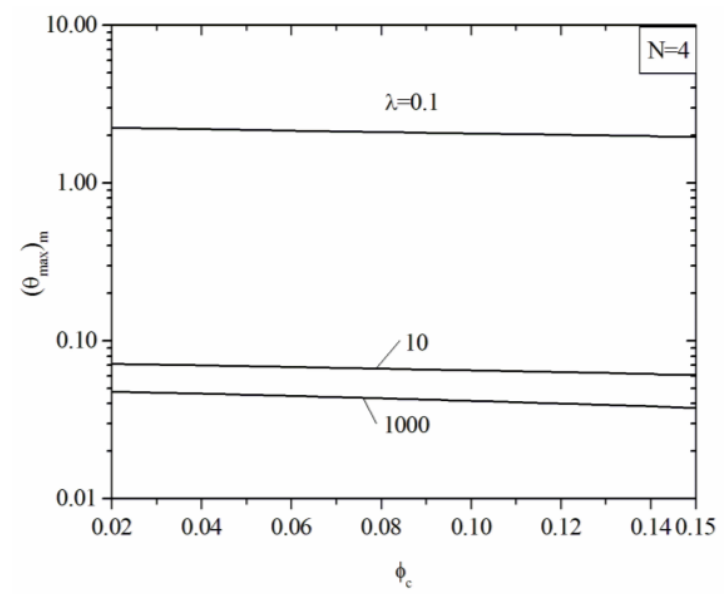

Figura 5: Efeito de $\phi_{c}$ sobre a temperatura máxima uma vez otimizada $\left(\theta_{\max }\right)_{m}$ para $N=4$ e diferentes valores do parâmetro $\lambda$.

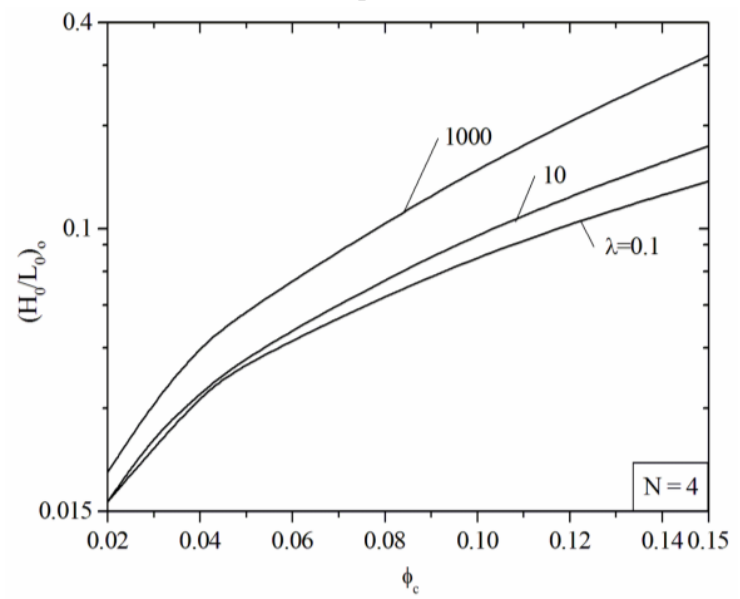

Figura 6: Efeito de $\phi c$ sobre a razão HO/LO uma vez otimizada para $N=4$ e diferentes valores do parâmetro $\lambda$.

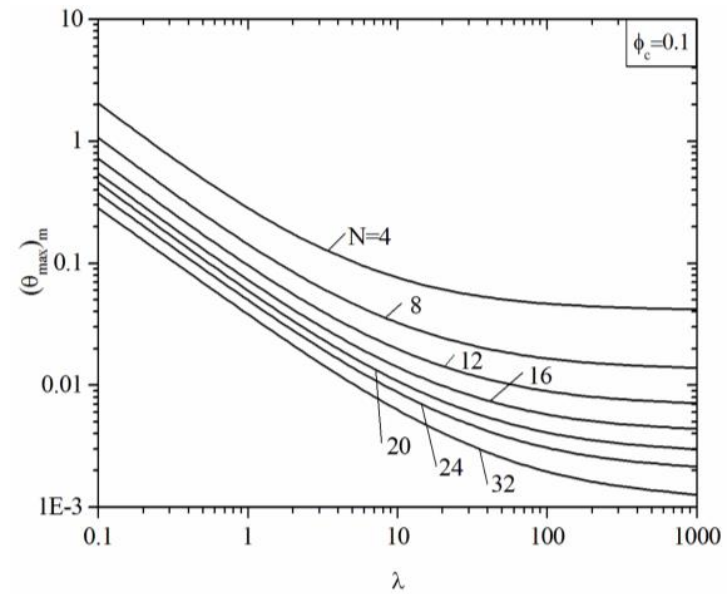

Figura 7: Efeito do parâmetro $\lambda$ sobre a temperatura máxima uma vez minimizada $\left(\theta_{\max }\right)_{m}$ para $\phi_{c}=0,1$ e diferentes valores de $N$. 


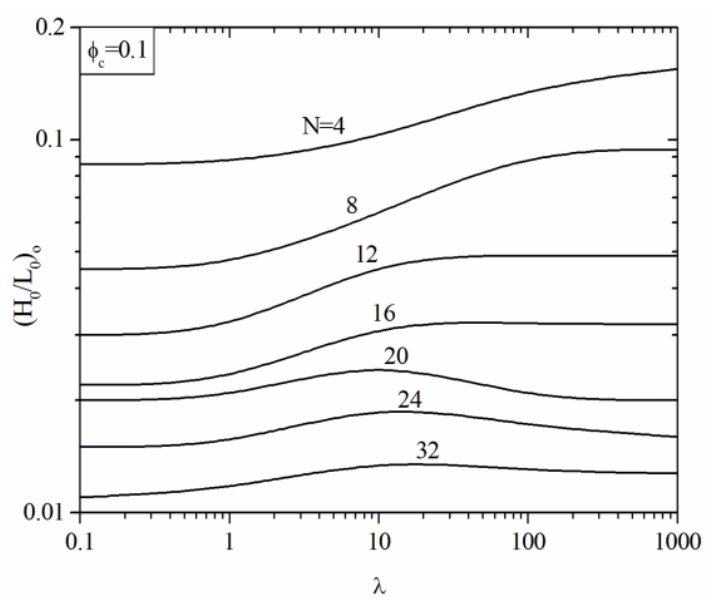

Figura 8: Efeito do parâmetro $\lambda$ sobre a razão uma vez otimizada $H_{0} / L_{0}\left(\theta_{\max }\right)_{m}$ para $\phi_{c}=0,1$ e diferentes valores de $N$.

Em geral, a Figura 7 apresenta uma diminuição de $\left(\theta_{\max }\right)_{\mathrm{m}}$ para os menores valores de $\lambda(0,1 \leq$ $\lambda \leq 10,0)$. Depois deste ponto, a taxa de redução é mais suave. Para os menores valores de $N$ uma estabilização do $\left(\theta_{\max }\right)_{\mathrm{m}}$ pode ser notado por $\lambda \geq 10,0$, mostrando que o aumento de $\lambda$ não vai levar a uma melhora do desempenho térmico do sistema. Em contrapartida, para os maiores valores de $N(N=24$ e 32$)$ a taxa de redução é suavizada, mas $\left(\theta_{\max }\right)_{\mathrm{m}}$ não estabiliza, mesmo no limite superior de $\lambda$ estudado. Os resultados indicaram que ainda é possível melhorar o rendimento térmico do sistema pelo aumento do parâmetro $\lambda$ para os casos com mais cavidades $(N=24$ e 32$)$.

A Figura 8 ilustra o efeito do parâmetro $\lambda$ sobre a fração ótima $\left(H_{0} / L_{0}\right)_{\circ}$ para vários valores de $N$. Para $N=4$ e $\lambda \leq 1.0$, a razão $\left(H_{0} / L_{0}\right)_{\text {o }}$ permanece constante. Depois deste ponto ocorre um aumento da razão $\left(H_{0} / L_{0}\right)_{0}$. Para $N=8,12$ e 16 o comportamento de $\left(H_{0} / L_{0}\right)_{0}$ como função de $\lambda$ tem uma ligeira mudança em comparação com o que observado para $N=4$. Para estes casos, foi observado uma estabilização da razão $\left(H_{0} / L_{0}\right)_{0}$ no extremo superior dos parâmetros $\lambda$ avaliados. Quando $N=20,24$ e 32, ocorre um aumento da razão $\left(H_{0} / L_{0}\right)_{0}$ até um limite superior de $\lambda=$ 10,0 e, a partir deste ponto, a razão $\left(H_{0} / L_{0}\right)_{0}$ diminui e estabiliza. É importante destacar que melhor desempenho térmico ao longo do domínio ocorre para valores altos do parâmetro $\lambda$. Este efeito é observado até um limite superior, no qual o melhor desempenho é alcançado, ou seja, de acordo com o princípio constructal da "distribuição ótima das imperfeições". Como consequência deste princípio, as formas ótimas variam mais intensamente quanto menor o valor de $\lambda$ e tendem a ficarem fixas quanto maior o valor de $\lambda$, já que a corrente nas cavidades alcança um limite superior de calor que pode ser removido pela parede do sólido.

\section{CONCLUSÃO}

Neste trabalho foi otimizada numericamente a forma de cavidades inseridas em um sólido cilíndrico. O número de cavidade variou de $N=4$ a 32, enquanto a fração de área ocupada pela cavidade mudou de $\phi_{c}=0,02$ a 0,15 .

Os resultados mostram que existe uma forma ótima $\left(H_{0} / L_{0}\right)_{\mathrm{o}}$ que minimiza a temperatura máxima, $\left(\theta_{\max }\right)_{\mathrm{m}}$, para cada fração da área da cavidade, o número de cavidades e parâmetro adimensional $\lambda$. Os resultados também mostram que para $\phi_{c}$ e $\lambda$, há um número ótimo de cavidades $(N)$ que minimiza a temperatura máxima e este número aumenta, em geral, a medida que $\phi_{c}$ e $\lambda$ também aumentam.

Os resultados estão de acordo com o princípio da distribuição ótima de imperfeições, ou seja, a evolução para configurações mais complexas faz com que ocorra uma redistribuição dos pontos de calor para melhorar a performance do sistema. 


\section{AGRADECIMENTOS}

Elizaldo D. dos Santos, Liércio A. Isoldi e Luiz A. O. Rocha agradecem ao CNPq pelo apoio ao trabalho.

\section{REFERÊNCIAS BIBLIOGRÁFICAS}

1. Bejan A, Zane JP. Design in Nature, New York, Doubleday, 2012.

2. Bejan A. Advanced Engineering Thermodynamics, 2nd ed., Wiley, New York, 1997.

3. Bejan A. Shape and Structure, from Engineering to Nature, Cambridge University Press, Cambridge, UK, 2000.

4. Bejan A, Lorente S. Design with Constructal Theory, Wiley, Hoboken; 2008.

5. Bejan A, Lorente S. Constructal Theory of Generation of Configuration in Nature and Engineering. Journal of Applied Physics. 2006;100: 041301.

6. Bejan A, Lorente S. The Constructal Law and the Evolution of Design in Nature. Phys. Life Rev. 2011;8:209-240, doi:10.1016/j.plrev.2011.05.010.

7. Bejan A, Lorente S. The Constructal Law of Design and Evolution in Nature. Phil. Trans. R. Soc. B 2010;365:1335-1347.

8. Bejan A, Lorente S. Constructal law of design and evolution: Physics, biology, technology and society. Journal of Applied Physics. 2013;113: 041301, doi:10.1063/1.4798429.

9. Bejan A, Charles JD, Lorente S. The evolution of airplanes. Journal of Applied Physics. 2014;116: 044901, doi:10.1063/1.4886855.

10. Bejan A, Badescu V, De Vos A. Constructal Theory of Economics Structure Generation in Space and Time. Energy Convers. Manage. 2000;41:1429-1451.

11. Beyene A, Peffley J. Constructal Theory, Adaptive Motion, and Their Theoretical Application to Low-Speed Turbine Design. J. Energ Eng-ASCE. 2009;135(4):112-118.

12. Kang D-H, Lorente S, Bejan A. Constructal Dentritic Configuration for the Radiation Heating of a Solid Stream. J. Appl. Phys. 2010;107:114910, doi:10.1063/1.3429195.

13. Kim Y, Lorente S, Bejan A. Constructal Multi-Tube Configuration for Natural and Forced Convection in Cross-Flow. Int. J. Heat Mass Transfer. 2010;53:5121-5128, doi:10.1016/j.ijheatmasstransfer.2010.07.053.

14. Kim Y, Lorente S, Bejan A. Steam Generator Structure: Continuous Model and Constructal Design. Int. J. Energy Res. 2011;35:336-345, doi: 10.1002/er.1694.

15. Azad AV, Amidpour M. Economic Optimization of Shell and Tube Heat Exchanger based on Constructal Theory. Energy. 2011;36:1087-1096, doi:10.1016/j.energy.2010.11.041.

16. Lee J, Kim Y, Lorente S, Bejan A. Constructal design of a comb-like channel network for self-heating and self-cooling. Int. J. Heat Mass Transfer. 2013;66:898-905, doi:10.1016/j.ijheatmasstransfer.2013.07.070.

17. Bejan A, Almogbel M. Constructal T-shaped fins, conducting body. Int. J. Heat Mass Transfer. 2000; 43:2101-2115.

18. Lorenzini G, Rocha LAO. Constructal design of Y-shaped assembly of fins. International Journal of Heat and Mass Transfer. 2006;49:4552-4557, doi:10.1016/j.ijheatmasstransfer.2006.05.019.

19. Lorenzini G, Rocha LAO. Constructal design of T-Y assembly of fins for an optimized heat removal. Int. J. Heat Mass Transfer. 2009;52:1458-1463, doi:10.1016/j.ijheatmasstransfer.2008.09.007.

20. Biserni C, Rocha LAO, Bejan A. Inverted fins: geometric optimization of the intrusion into a conducting wall. Int. J. Heat Mass Transfer. 2004;47:2577-2586, doi:10.1016/j.ijheatmasstransfer.2003.12.018.

21. Xie Z, Chen L, Sun F. Geometry optimization of T-shaped cavities according to constructal theory. Math. Comput. Model. 2010;52:1538-1546, doi:10.1016/j.mcm.2010.06.017.

22. Lorenzini G, Biserni C, Isoldi LA, Dos Santos ED, Rocha LAO. Constructal Design Applied to the Geometric Optimization of Y-shaped Cavities Embedded in a Conducting Medium. J. Electronic Packaging. 2011;133(40):041008-1 - 041008-8, doi:10.1115/1.4005296.

23. Xie ZH, Chen LG, Sun SF. Constructal optimization of twice Y-shaped assemblies of fins by taking maximum thermal resistance minimization as objective. Sci. China Tech Sci. 2010;53(10):2756-2764, doi: 10.1007/s11431-010-4037-x.

24. Biserni C, Rocha LAO, Stanescu G, Lorenzini E. Constructal H-shaped cavities according to Bejan's theory. Int. J. Heat Mass Transfer. 2007;50: 2132-2138, doi:10.1016/j.ijheatmasstransfer.2006.11.006.

25. Lorenzini G, Rocha LAO. Geometric optimization of T-Y-shaped cavity according to Constructal design. Int. J. Heat Mass Transfer. 2009;52: 4683-4688, doi:10.1016/j.ijheatmasstransfer.2009.06.020. 
26. Lorenzini G, Rocha LAO, Biserni C, Dos Santos ED, Isoldi LA. Constructal design of cavities inserted into cylindrical solid body. Journal of Heat Transfer. 2012;134(7): 071301, doi: $10.1115 / 1.4006103$.

27. Rocha LAO, Lorente S, Bejan A. Vascular design for reducing hot spots and stresses. Journal of Applied Physics. 2014; 115: 174904, doi: 10.1063/1.4874220

28. MATLAB. User's Guide, Version 6.0.088, Release 12. 2000, The Mathworks Inc., Natick, MA.

29. See www.comsol.com to consult the user manual. 